doi: http://dx.doi.org/10.7124/bc.0009E4

\section{H-2. Lamin plays with SUN}

\section{Lenka Hůlková $^{1}$, Jana Rohožková ${ }^{1}$ and Pavel Hozák ${ }^{1,2}$}

${ }^{1}$ Institute of Molecular Genetics ASCR, v.v.i. division BIOCEV; Dept. of Epigenetics of the Cell Nucleus, Vestec, Czech Republic; ${ }^{2}$ Institute of Molecular Genetics ASCR, v.v.i., Lab. of Biology of the Cell Nucleus, Prague, Czech Republic lenka.hulkova@img.cas.cz

Intermediate filaments (IFs) are cytoskeletal components composed of a family of related proteins sharing common structural and sequence features. Beside cytoplasmic IFs, we know about the nuclear IFs - lamins, which belong to this protein family as a type V. Lamins are organized at the nuclear periphery, where form the main component of nuclear lamina and participate in many roles, such as maintenance of nuclear shape, regulation of gene expression, transcription, DNA replication, segregation of chromosome, meiosis and apoptosis. Mutations in lamin genes have a dramatic effect on their function causing pathological phenotype (i.e. Emery-Dreifuss Muscular Dystrophy (EDMD) or HutchinsonGilford Progeria Syndrome (HGPS)). We identified a novel mechanism of lamin involvement in the process of gametogenesis in Caenorhabditis elegans. This model organism offers an advantage of only one evolutionarily conserved lamin protein (CeLMN) compared to the mouse or human cell lines. Our project focuses on the molecular mechanisms and regulation of chromosomal dynamics during gametogenesis. We study the SUN/ KASH complex, which is important in the process of chromosome pairing at early stag- es of meiosis I. Interestingly, we discovered that some dedicated mutations in CeLMN gene (which mimic EDMD) affect dynamics of SUN/KASH complex. Subsequently, this change in properties of SUN/KASH complex causes defects in development of germ cells in C. elegans. Based on our results, we suggest a new possible model for the functional connection of SUN/KASH complex to the nuclear lamina.

Supported by the project „BIOCEV”(CZ.1.05/1.1.00/02.0109) and "Modernization and support of research activities of the national infrastructure for biological and medical imaging Czech-Biolmaging" (No. CZ.02.1.01/0.0/0.0/16 013/0001 775) funded by the European Regional Development Fund, by GACR (16-03403S and 18-19714S) and UMG/RVO: 68378050 . We acknowledge the Ministry of Education, Youth and Sports of Czech Republic COST Inter-excellence internship program LTC17.

doi: http://dx.doi.org/10.7124/bc.0009E5

\section{K-1. Telomere biology in the Drosophila germline: link between chromatin structure, transcription and nuclear localization}
A. Kalmykova $^{1}$, V. Morgunova ${ }^{1}$, A.Y. So- lovyova ${ }^{1,2}$
${ }^{1}$ Institute of Molecular Genetics, Russian Academy of Sciences, Moscow 123182, Russia; ${ }^{2}$ Department of Biochemistry, Faculty of Biology, Lomonosov Moscow State University, 119991 Moscow, Russia allakalm@img.ras.ru

Telomeres are nucleoprotein complexes that protect eukaryotic linear chromosome ends. They are composed of the telomeric DNA and 
proteins associated with telomeric repeats. Transcription of telomeric repeats is a conserved feature revealed in many species, and the mechanisms of the telomere homeostasis are closely related to regulation of the telomeric repeat transcription. The telomeres of Drosophila are maintained in the absence of telomerase by the transpositions of the specialized telomeric retrotransposons. Using the Drosophila model, we study the relationship between the telomeric chromatin state and telomeric repeat expression in the female germline. In this study, we have examined the changes in the telomeric chromatin structure and nuclear telomere positioning induced by massive overexpression of telomeric repeats using RNA/DNA fluorescence in situ hybridization, immunostaining and chromatin immunoprecipitation methods. Heterochromatin protein 1 (HP1) and H3K9me3 are important components of telomeric chromatin in different species; however, the mechanisms underlying their deposition at the telomere are not clear. Disruption of the PIWI-interacting RNA pathway causes overexpression of telomeric repeats, a loss of heterochromatic components, and translocation of telomeres from the periphery to the nuclear interior. However, piRNAs are not required either for assembly of the protective telomere capping complex or for telomere clustering in the germline. We have revealed that piRNAs provide a germline-specific mechanism for HP1, Rhino, a germlinespecific HP1 homolog, and H3K9me3 deposition at telomeric retrotransposon arrays, thus ensuring telomere silencing at the transcription level during gametogenesis. It has also been found that telomeric transcript levels are regulated by the factors of co-transcriptional RNA surveillance, such as the deadenylase Ccr4-Not complex and nuclear RNA-binding protein Ars2. Noteworthy, the mechanisms for nuclear metabolism of telomeric transcripts are highly conserved. Indeed, Ars2 suppresses transcription of telomeric repeats both in human cells and in Drosophila. Depletion of the factors of co-transcriptional RNA metabolism results in the accumulation of Drosophila telomeric transcripts at transcription sites without the loss of heterochromatin marks. In this case, telomeres remain localized to the nuclear periphery. Taken together, these data indicate that transcription and chromatin states of telomeres rather than accumulation of chromatin-associated telomeric transcripts determine a peripheral localization of telomeres in the Drosophila germline.

Russian Foundation for Basic Research (19-04-00254)

doi: http://dx.doi.org/10.7124/bc.0009E6

\section{K-2. Role of ERK 1/2 pathway in polyploidization of hepatocytes in cholestatic liver}

S. Kiparoidze, E. Bakuradze, I. Modebadze, D. Dzidziguri, K. Agagulian

Department of Biology, Iv. Javakhishvili Tbilisi

State University, Tbilisi, Georgia

salo_kipa@yahoo.com

In response to resection, the importance of cell proliferation, polyploidization and hypertrophy for liver regeneration and their contribution to rapid recovery of functions is well studied. However, relatively little information exists about liver regeneration 